\title{
Concrete beam bending test monitorization using a high strain fiber optic sensor
}

\author{
M. Bravo, J. Sáenz, M. Bravo-Navas and M. Lopez-Amo Senior Member, IEEE, Member, OSA
}

\begin{abstract}
In this work the development of an "eight-shape" fiber optic sensor for high strain measurement is experimentally demonstrated. This strain sensor is interrogated using a commercial optical time domain reflectometer through a dual configuration that is able to measure backscattering and the transmission of an intensity pulse while reducing unwanted cladding-mode coupling effects. An 'in field' concrete beam bending test was also performed in order to compare the proposed low-cost intensity bending sensor with the mature fiber Bragg grating technology.
\end{abstract}

Index Terms- OTDR, fiber Bragg grating, structural health monitoring, bending sensors.

\section{INTRODUCTION}

I $\mathrm{n}$ the past years, a considerable amount of research activity has been focused on the development of what is commonly called "smart structures". These "smart structures" have the ability of providing real-time information about their health and integrity and sometimes they can even adapt themselves to compensate any anomalous in-service or environmental loading, or other potentially structural-weakening conditions. The first step in order to develop this technology is to incorporate a structural sensing capability. This can be achieved, for example, through the use of strain measurement units at multiple locations throughout the structure [1].

Fiber Bragg grating (FBGs) have become a dominant fiber sensing tool. These wavelength selective all-fiber devices have undergone a rapid development following the observation of a very-narrow-band reflection in the photosensitive core region of Ge-doped silica optical fibers [2] and its first successful fabrication on a fiber core by exposure of a coherent two-beam UV interference pattern in 1989 [3]. FBGs have all the advantages of fiber optic technology: they are immune to electromagnetic interferences (EMI), they are lightweight and have small physical dimensions, which makes them suitable for embedding into or attach to a structure. No external wires are required to connect these sensors to the control system as the fibers themselves act as both the sensing element and the signal

\footnotetext{
Manuscript received June 30, 2011.

The authors are grateful to the Spanish Government project TEC201020224-C02-01.

M. Bravo and M. López-Amo are with the Departamento de Ingeniería Eléctrica y Electrónica of Universidad Pública de Navarra, Campus de Arrosadía, 31006, Pamplona, Navarra, Spain

J. Sáenz and M. Bravo-Navas are with Eurocontratas S.A. C/ El Carmen, 1$1^{\circ}, 26005$ Logroño, La Rioja, Spain

(Corresponding e-mail: mikel.bravo@unavarra.es).
}

propagation conduit. FBG sensors offer the advantage of single ended connection to control systems because only the reflected signals from the FBGs are important for demodulation. FBGs possess excellent resolution and range, water and corrosion resistance, the ability to be multiplexed, immunity to harsh weather conditions, compact sensor size, and reasonable cost per channel [4]. The technical specifications of the FBG strain sensor used for this paper are the following: $1.2 \mathrm{pm} / \mu \varepsilon$ sensitivity, $4000 \mu \varepsilon$ measurement range, $\pm 2 \mu \varepsilon$ accuracy and $1 \mu \varepsilon$ resolution [5]. Also the manufacturer has informed us they have tested the FBG in a range of $25 \mathrm{~nm}$, which corresponds to 21 milli-strain, being this the breaking point. The range of the sensor is not an absolute value, but is related to the cumulative probability of failure and fatigue and reliability also depends on the fiber stripping techniques are used. [6]. These $4000 \mu \varepsilon$ measurement range corresponds to a typical value of the maximum strain applicable to FBGs and valid for most part of applications. However, specially designed FBGs can reach up to 10000 micro-strain [7]

An alternative for FBGs in high strain applications are optical fiber intensity sensors based on bend mechanisms, that have been extensively used in different applications [8]-[10]. They are simple, reliable and low-cost elements that can be also multiplexed into a sensor network. Optical time domain reflectometer (OTDR) has become a valuable instrument to interrogate this kind of sensors, by measuring the induced changes in the optical intensity at different locations in the fiber [11].

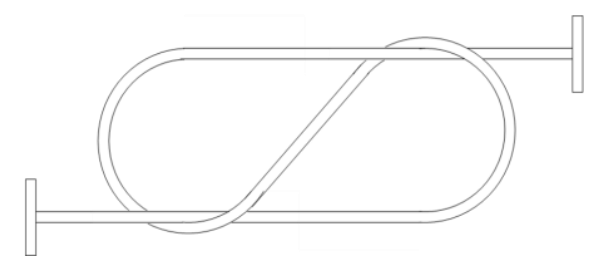

Fig. 1. High strain "eight-shape" sensor shape.

In this paper, we present the application of a simple "eightshape" fiber optic bending sensor for high strain monitorization. A concrete beam bending test is also presented. This test has been monitored simultaneously using FBG sensors and the "eight-shape" intensity sensor. This intensity sensor consists of a fiber tied in the shape of an eight number, which was first presented in 1997 by Sienkiewicz et al [12]. These sensors were interrogated by a fiber Bragg grating interrogator and an OTDR [13] until the break of the concrete beam. 
The concrete beam designed to the bending test was made by a HA25/P/20/2B concrete (Spanish normative) this means that this is a reinforced concrete with $25 \mathrm{Nw} / \mathrm{mm}^{2}$ of plastic consistency, 20mm of maximum gravel size and was designed for normal external weather conditions. The reinforcement was made by 8 steel beams of $16 \mathrm{~mm}$ diameter placed as shown in section III. The beam dimensions were $6 \mathrm{~m}$ length, $0.5 \mathrm{~m}$ height and $0.3 \mathrm{~m}$ width. As bending test, a four point test was performed. The beam was placed on a testing bench on two points located $25 \mathrm{~cm}$ from the ends and the load was applied at two different points for achieving an uniform bending moment in the center of the beam. Therefore, the tensile stress was suffered at the bottom and the compressive stress at the top. These areas of the beam are considered ideal for testing [14].

\section{SENSOR DEVELOPMENT}

The high strain intensity sensor used in this work consists of a standard single mode fiber "tied" into the shape of a figure of eight, as it is shown in Fig. 1. When a displacement in length occurs, the sensing head size decreases and a power loss is induced through the decrease of the loop radius [8]. This fiber structure, properly bonded, can be used as a high strain sensor. The setup used is presented in Fig. 2, where the backscattering and the transmitted power are simultaneously detected by a commercial OTDR EXFO FTB-7423B-B.

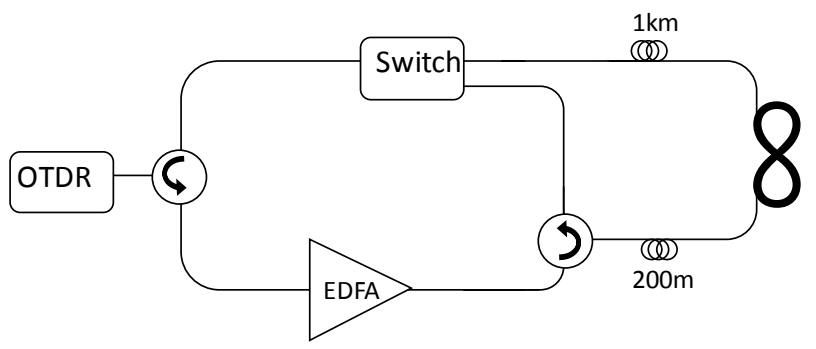

Fig. 2. Backscattered and transmission setup proposed to avoid claddingmodes coupling.

Two circulators, an optical switch and an EDFA are used. The launched pulses and the backscattered signal reaching the OTDR are physically separated using a first circulator. The launched pulses are amplified by an EDFA, in order to compensate the loss of the remaining components and to increment the dynamic range in the OTDR. The switch is used to select the transmission pulses that go through the "eightshape" sensor or the backscattered power redirected by the circulator located after the EDFA. Finally, both, backscattering and transmission acquired traces are processed and combined. Figure 3 presents the experimentally obtained backscattering and transmission data of the proposed sensor.

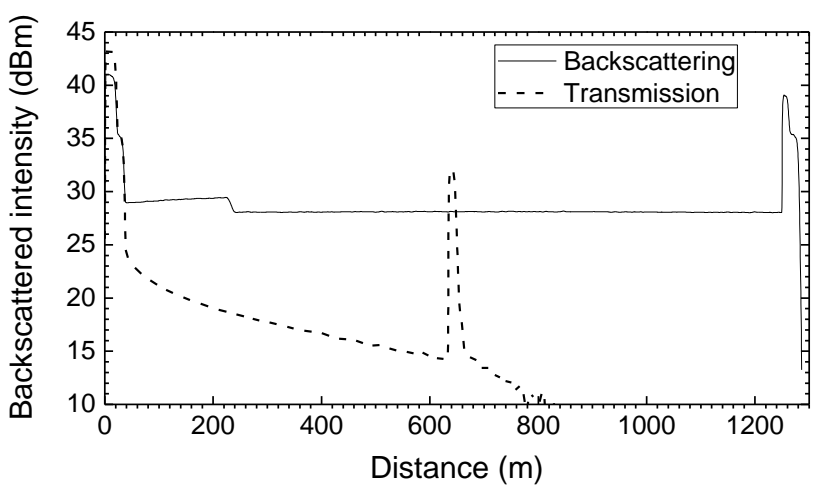

Fig. 3. Backscattering and transmission traces obtained by the OTDR.

The processing method involves the calculation of the mean backscattered power of both OTDR traces in the measuring zone which changes with the displacement. In the backscattered trace we have used the measured values corresponding to the zone between $200 \mathrm{~m}$ and $1250 \mathrm{~m}$, and in the transmission trace, the points used are the corresponding to the peak. This peak appears at half of the distance of the network's length because the OTDR measuring software divides by two the propagation time along the tested set-up. Because in this transmission measurement the light only travels one way, the OTDR understands that the detected signals are "reflected" at half of the distance. Finally the result for each trace is added. The reason to combine both traces comes from the observation of the transmitted signal and the backscattered signal cladding-modes. The behavior of traces corresponding to both signals is, for some bending radius, complementary.

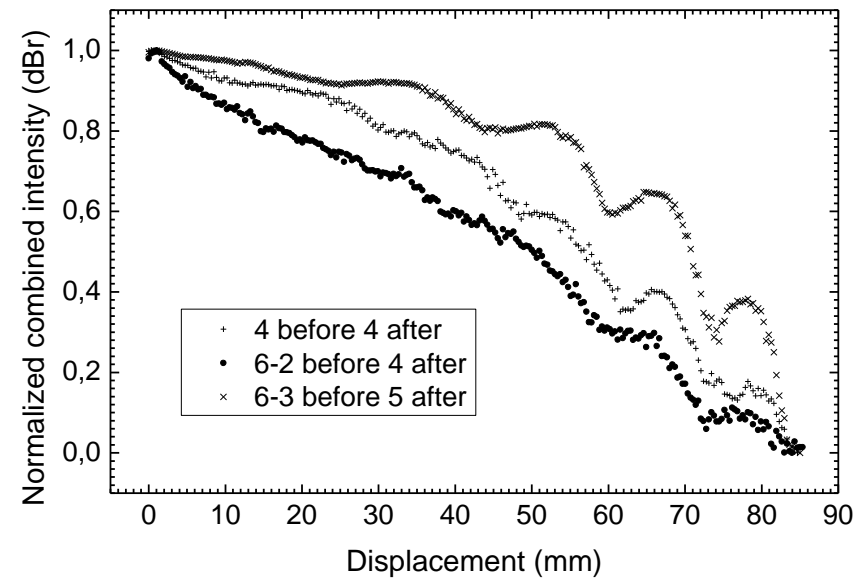

Fig. 4. Received power for different loops configurations versus displacement.

This processing technique combined with a cladding mode recombination before and after the "eight-shape" sensor is necessary to achieve a good sensor behavior. For this task various loop diameters of single-mode fiber have been tested to achieve the best bending response. In order to avoid cladding-mode coupling, additional loops acting as mode scramblers were used before and after the "eight-shape" sensor. These mode scramblers radiate the cladding modes 
before and after the sensor to achieve the most linear response. Different diameter fiber loops were tested as shown in fig. 4. The different number of loops was chosen empirically and, as is shown in fig. 4, a small change in the number of loops in this configuration changes the response significantly. For this reason it is important to calibrate this parameter before use this sensor system. In this case the best result was achieved using 6 loops of $35 \mathrm{~mm}$ of diameter- into the sensors, $2 \times 25 \mathrm{~mm}$ loops before the "eight-shape" and $5 \times 35 \mathrm{~mm}$ loops after the "eight-shape" as mode scramblers.

\section{RESULTS AND DISCUSSION}

The previous presented sensors were identified as suitable for high strain measurements, in applications where FBGs suffer excessive deformation and finally, break. In order to demonstrate their suitability, an experiment was designed to collect different data from different sensors in order to verify the behavior of the sensors in concrete structures. Different sensors have been used in a concrete beam bending test: embedded FBG strain and temperature sensors, surface FBG strain sensors and the "eight-shape" strain sensor. FBG sensors were interrogated by a SM125 interrogation unit from Smartech and the "eight-shape" strain sensor was interrogated by the EXFO OTDR unit. In Figure 5 the different sensors used are shown, and in Figure 6 the setup used to interrogate the "eight-shape" sensor is presented. In this case only the OTDR is used for measurements of a $1200 \mathrm{~m}$ section of single mode fiber SMF28 with the "eight-shape" sensor located at $200 \mathrm{~m}$ from the OTDR.

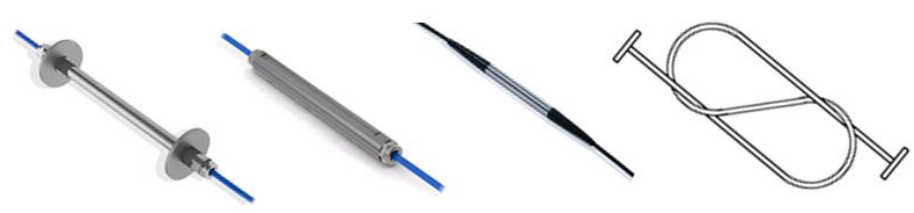

Fig. 5. Strain and temperature FBG embedded sensors from Fiber Sensing and the "eight-shape" sensor.

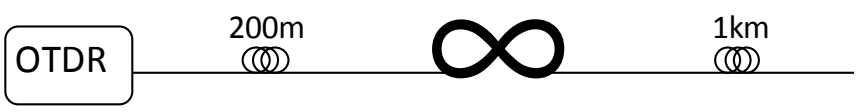

Fig. 6. High strain sensor setup used during the bending test.

The embedded FBG sensors were installed in the concrete beam before the pouring. In Figure 7 the beam transversal section with the sensor distribution can be seen. The external strain FBG and the "eight-shape" sensor were installed before the bending test. This figure also depicted the steel beams distribution as circles $(4 \varnothing 16 \mathrm{~mm}$ means 4 steel beams of $16 \mathrm{~mm}$ diameter). An external temperature FBG sensor was operative during all the experiment. Fig. 8 shows the sensors distribution and the different beam dimensions. Figure 8 also shows the four point bending test set-up. The triangles at the bottom of the beam correspond to the beam support points and the arrows in the top of the picture indicate the points where the load is applied (shown as $\mathrm{P} / 2$ ).

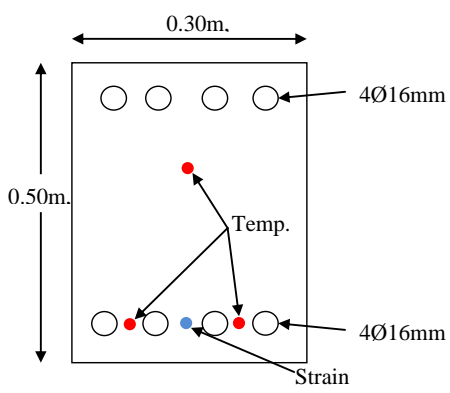

Fig. 7. Beam transversal section showing the sensors distribution. Temp: FBG temperature sensors. Strain: FBG Strain sensor. $4 \varnothing 16 \mathrm{~mm}$ : 4 steel beams of $16 \mathrm{~mm}$ of diameter).

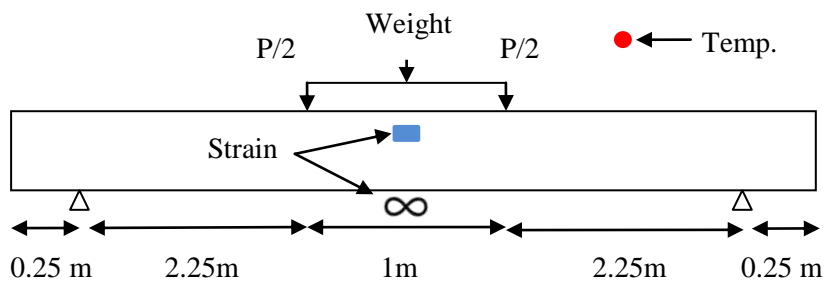

Fig. 8. Beam longitudinal view with the sensors distribution and the bending test configuration setup

TABLE I

APPLIED LOAD, DEFORMATION AND EVENTS DURING THE TEST

\begin{tabular}{lll}
\hline \hline Load $(\mathrm{kg})$ & $\begin{array}{l}\text { FBG strain } \\
\text { (micro-strain) }\end{array}$ & Event \\
\hline 500 & 46,79 & \\
1000 & 59,81 & \\
1500 & 77,77 & \\
2000 & 103,01 & \\
2500 & 153,21 & \\
2750 & 229,14 & \\
3000 & 281,03 & \\
3500 & 341,20 & \\
4000 & 772,94 & \\
4500 & 979,34 & \\
5000 & 1105,22 & \\
6000 & 1315,94 & \\
7000 & 1533,97 & \\
8000 & 1723,89 & \\
9000 & 1907,98 & \\
10000 & 2050,90 & \\
11000 & 2196,63 & \\
12000 & 2334,25 & \\
13000 & 2430,90 & \\
14000 & 2550,53 & \\
15000 & 2675,40 & Beam breackage \\
16000 & 2922,86 & \\
17000 & 3153,41 & \\
17000 & 3320,06 & Steel plastic deformation \\
17000 & 3890,07 & \\
\hline \hline
\end{tabular}


After all sensors were installed and prepared for the bending test, different weights were applied in the middle of the concrete beam. As Table 1 indicates, initially, 500 kilograms steps were applied from the start to the first crack, which appeared when reaching an applied weight of $\sim 4500$ kilograms. After this crack appeared, 1000 kilograms weight increments were applied until the break of the beam. After this point the steel comes at a state of plastic deformation which indicates that the beam does not bear more load. We introduced more deformation until the breakage of the FBG. Figures 9 and 10 show pictures taken of the pole structure before and after the bending test, respectively.

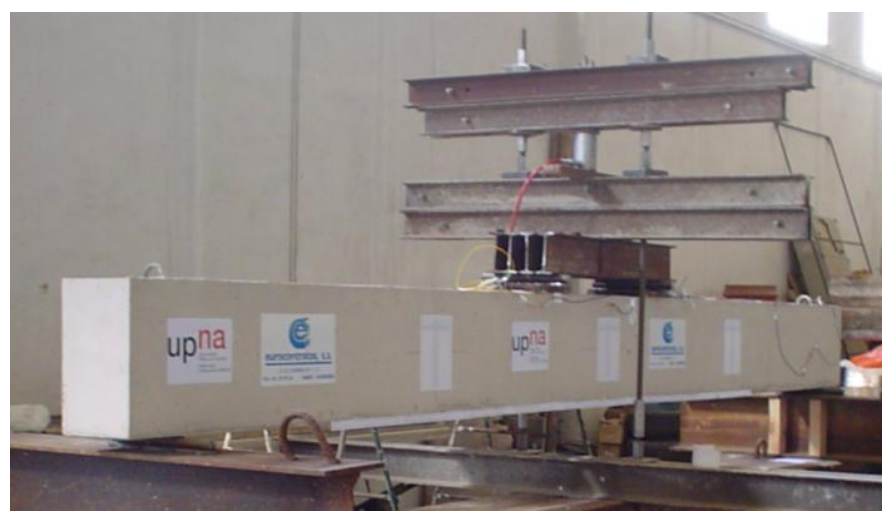

Fig. 9. Pre bending test beam picture

Both, concrete embedded FBG and "eight-shape" sensors monitored the concrete beam behavior during the bending test. The plots in fig. 11 and fig. 12 show the strain measurements as a function of time and the weight applied to the beam, respectively. The FBG strain vs. time trace is visible during all the events in the bending test: the cracking point, all the weight steps and when the FBG broke. The FBG measurement range was overcome in about 1000 micro-strain above the company specifications. FBG pre-break measurements that exceeded this range cannot be considered as reliable. In both plots, OTDR-“eight-shape" strain sensor results are also shown.

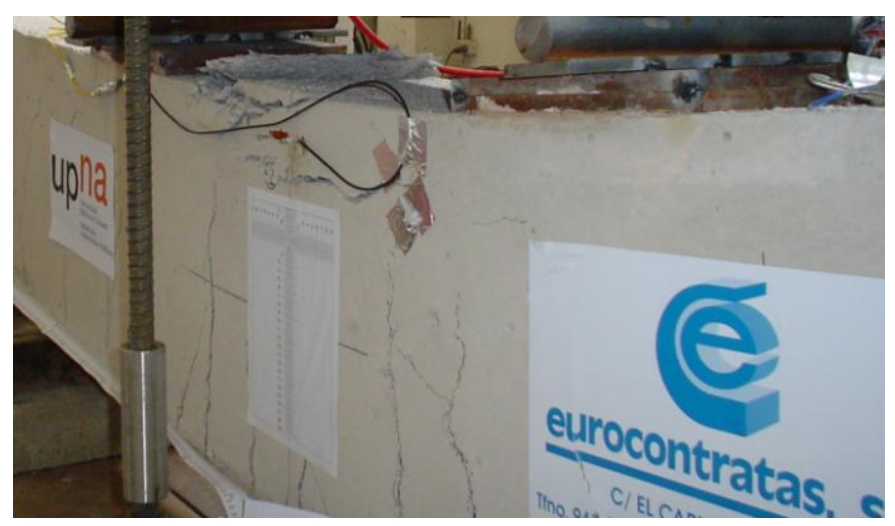

Fig. 10. Post bending test beam picture

These "eight-shape" sensor results adjust correctly to the accurate FBG results. We would like to remark that the low cost "eight-shape" sensor has a higher measurement range than the utilized FBG. The figure 12 plot shows two different events, the cracking point at $12: 15 \mathrm{~h}-4000 \mathrm{~kg}$, and the failure point at $13: 50 \mathrm{~h}-17000 \mathrm{~kg}$. The strain measurement at failure is significantly high. This strain increment, due to the overstrained steel could be measured only by the "eightshape" sensor. Its demonstrated measuring range is three times higher than FBGs one.Passed 1 hour and 30 minutes of test session (see Fig. 11) and applying a weight of $12000 \mathrm{Kg}$ some deviant points appeared due to cladding-modes coupling. In order to avoid cladding-modes coupling, the above mentioned fiber mode scramblers before and after the tie strain sensor were developed.

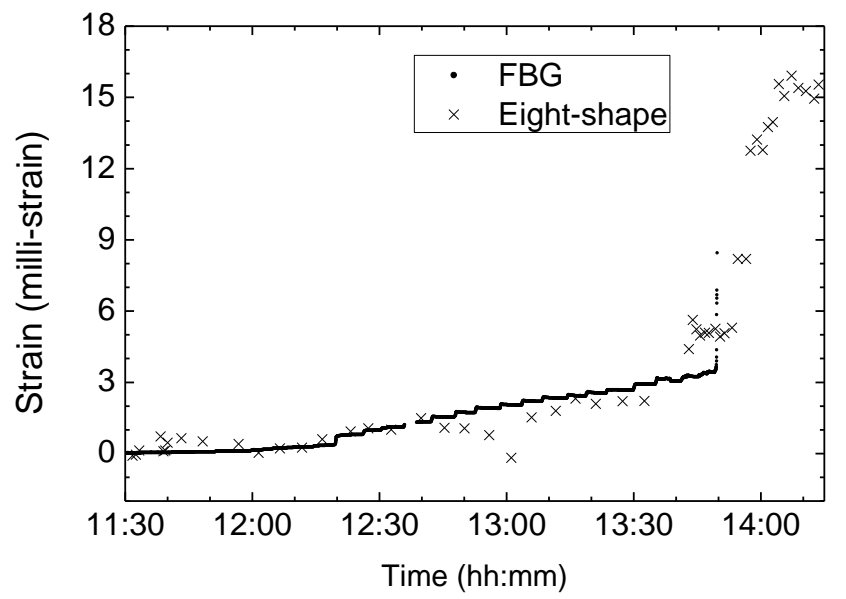

Fig. 11. Measured strain by the inner FBG and the "eight-shape" sensor during the bending test.

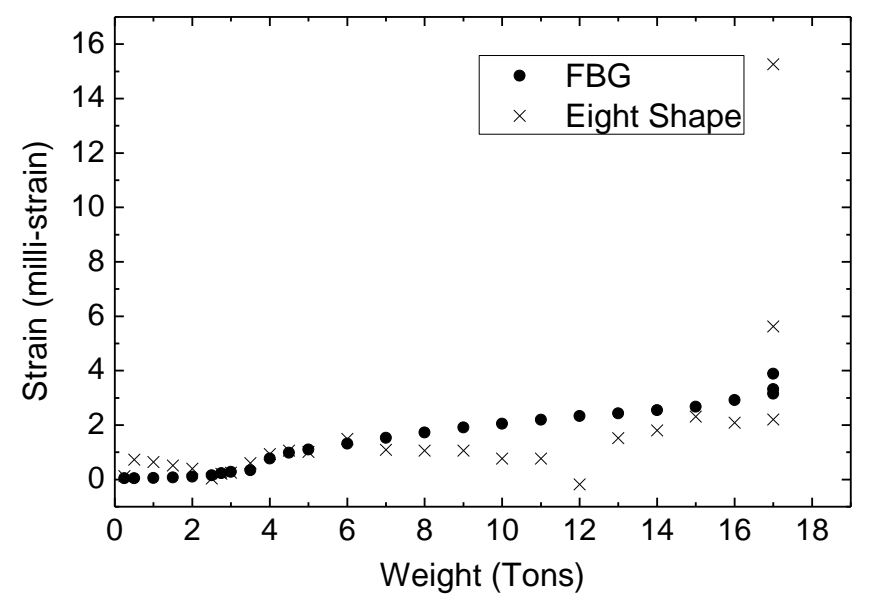

Fig. 12. Measured strain by the inner FBG and the "eight-shape" sensor versus the applied weight.

\section{CONCLUSIONS}

In this paper the application of low cost bending sensor for high strain measurements has been proposed and demonstrated. A concrete beam bending test has been monitored by FBGs and the low-cost intensity strain bending sensor proposed. The ability for high strain measurement of the proposed sensor was demonstrated during the bending test, showing a wider range of operation than the FBG. Also, the 
proposed setup improves the sensor system behavior reducing the unwanted cladding-mode coupling effects.

\section{REFERENCES}

[1] M. A. Davis, D. G. Bellemore and A. D. Kersey, "Distributed fiber Bragg grating strain sensing in reinforced concrete structural components," Cement and Concrete Composites, vol. 19, pp. 45-57, 1997.

[2] B. S. Kawasaki, K. O. Hill, D. C. Johnson and Y. Fujii, "Narrow- band Bragg reflectors in optical fibers." Opt. Lett.,vol. 3, pp. 66-68, 1978.

[3] G. Meltz, W. W. Morey and W. H. Glenn, "Formation of Bragg gratings in optical fibers by a transverse holographic method," Opt. Lett., vol. 14, pp. 823-825, 1989.

[4] M. Majumder, T. K. Gangopadhyay, A. K. Chakraborty, K. Dasgupta and D. K. Bhattacharya, "Fibre Bragg gratingsin structural health monitoring-Present status and applications," Sensors and Actuators A: Physical, vol. 147, pp. 150-164, 9/15, 2008.

[5] www.fibersensing.com

[6] P. Lefebvre, A. Vincelette, P. Ficocelli, S. Allard, S. Carbonneau, "Reliability characterization of fiber Bragg grating", Proc, Optical Fiber Sensors, OSA Technical Digest (CD) (OSA), paper MD6, 2006

[7] www.fos-ta.com

[8] J. W. Berthold III, "Historical review of microbend fiber-optic sensors," J. Lightwave Technol., vol. 13, pp. 1193-1199, 1995.

[9] N. Lagakos, J. H. Cole and J. A. Bucaro, "Microbend fiber-optic sensor," Appl. Opt., vol. 26, pp. 2171-2180, 1987.

[10] D. Dsignonlagić and M. Završnik, "Fiber-optic microbend sensor structure," Opt. Lett., vol. 22, pp. 837-839, 1997.

[11] N. M. P. Pinto, O. Frazão, J. M. Baptista and J. L. Santos, "Quasidistributed displacement sensor for structural monitoring using a commercial OTDR," Optics and Lasers in Engineering, vol. 44, pp. 771778, 2006.

[12] F. Sienkiewicz and A. Shukla, "A simple fiber-optic sensor for use over a large displacement range," Optics and Lasers in Engineering, vol. 28, pp. 293-304, 1997.

[13] M. Bravo, M. Fernandez-Vallejo and M. Lopez-Amo, "Hybrid OTDRfiber laser system for remote sensor multiplexing," Proc. SPIE, $4^{\circ}$ European Workshop on Optical Fibre Sensors, vol. 7653, 765340-1-4, 2010.

[14] W. K. Szaroletta, N. L. Denton, "Four Point Bending: A New Look" Proc. 2002 American Society for Engineering Education Annual Conference \& Exposition, Session 2168, 2002.
Mikel Bravo was born in Deba, Guipuzcoa, Spain, in June 1983. He received the Telecommunication Technical Engineering and Communications Master degrees from the Universidad Pública de Navarra, Spain in 2007 and 2010, respectively.

In 2008, he joined the Optical Communications Group, Department of Electrical and Electronic Engineering from Universidad Pública de Navarra. His research interests are in structural health monitoring, fiber Bragg gratings, intensity bending sensors, multiplexing architectures, optical amplifiers, and optical networks.

Manuel Lopez-Amo (M'91, SM '98) was born in Madrid, Spain, in 1960. He received the telecommunications engineering degree and $\mathrm{Ph} . \mathrm{D}$. degrees from the Universidad Politécnica de Madrid, Spain in 1985 and 1989, respectively. From 1985 to 1996, he belonged to the Photonic Technology Department of the Universidad Politécnica de Madrid, where in 1990 he became an associate professor. He has been a visiting researcher at INTEC of the University of Ghent, Belgium and at British Telecom Research Laboratories (Ipswich, U.K.).

In 1996, he moved to the Public University of Navarra (Pamplona, Spain) where he became a Full Professor in the Electrical and Electronic Engineering department and he is currently the head of the optical communications group of this department. He has been Chairman of the Optoelectronic Committee of Spain. He has been leader of more than 20 research projects and he has coauthored more than 150 works in international refereed journals and conferences related with fiber-optic networks, optical amplifiers, fiber-optic sensors, and integrated optics. He is a member of the technical committees of the International Conference on fiber optic sensors (OFS), the European Workshop on optical fiber sensors (EWOFS), and European COST TD 1001 action, among others. Professor López-Amo is senior member of the IEEE and member of the OSA 